\title{
Coexistence dans un réseau psycho-medico-social : apport de la théorie de la justification
}

\section{Isabelle Deliège}

\section{(2) OpenEdition}

1 Journals

Édition électronique

URL : http://journals.openedition.org/communicationorganisation/2898

DOI : 10.4000/communicationorganisation.2898

ISSN : $1775-3546$

Éditeur

Presses universitaires de Bordeaux

Édition imprimée

Date de publication : 1 mai 2004

ISSN : 1168-5549

Référence électronique

Isabelle Deliège, «Coexistence dans un réseau psycho-medico-social : apport de la théorie de la justification », Communication et organisation [En ligne], 24 | 2004, mis en ligne le 27 mars 2012, consulté le 03 mai 2019. URL : http://journals.openedition.org/communicationorganisation/2898 ; DOI : 10.4000/communicationorganisation.2898

Ce document a été généré automatiquement le 3 mai 2019.

(c) Presses universitaires de Bordeaux 


\title{
Coexistence dans un réseau psycho- medico-social : apport de la théorie de la justification
}

\author{
Isabelle Deliège
}

1 Si la question de la coexistence se pose dans une organisation, elle se pose avec plus d'insistance encore dans le cadre de collaborations entre différentes organisations et donc dans un réseau d'organisations. Aujourd'hui, le domaine psycho-médico-social est appelé à fonctionner de plus en plus de cette manière, en raison de la réalité à laquelle il doit faire face. Lorsque les problèmes s'accumulent, tant sur le plan relationnel, psychologique, médical, scolaire, que financier, dans une famille, les intervenants psychomédico-sociaux mobilisés tendent également à se multiplier. Or, ils sont généralement chacun attachés à une institution particulière : centre de santé mentale, hôpital, crèche, école. Centre Public d'Aide Sociale, pour ne donner que quelques exemples.

Différents intervenants sont donc parfois amenés à coexister dans une situation problématique auprès d'une famille, à tenir compte de la présence et des actions des uns et des autres, voire à travailler ensemble. Mais les choses ne sont pas simples: non seulement une multitude de professionnels peuvent se côtoyer, mais en plus, les divers organismes psycho-médico-sociaux actifs dans la situation remplissent généralement des missions différentes, complémentaires, mais parfois aussi concurrentes. En outre, ces dernières années, on assiste dans ce domaine à un phénomène particulier: ces organisations tendent de plus en plus à se structurer en réseau. - il est souvent question d'«intervention de réseau», de "travail en réseau» - ce qui incite à penser la coexistence dans un tel réseau et ses modalités pratiques.

3 Nous commencerons par poser la question de la coexistence dans le cadre d'un réseau d'organisations dans le domaine psycho-médico-social ${ }^{1}$. Ensuite, nous verrons comment l'apport de la théorie de Boltanski et Thévenot ${ }^{2}$, basée sur une pluralité de mondes, permet d'envisager cette problématique. Enfin, nous terminerons en présentant un de ces 
mondes, basé sur la notion de projet ${ }^{3}$ et lié à celle de réseau, ce qui nous semble particulièrement intéressant pour la compréhension du travail en réseau.

\section{La question de la coexistence dans un réseau d'organisations du domaine psycho-médico-social}

4 Le champ psycho-médico-social est aujourd'hui, tout comme le monde de l'entreprise, confronté à une mise en réseau des entités qui le composent. Différentes raisons peuvent être avancées pour expliquer cet état de l'ait: l'accumulation de problèmes d'ordres divers dans les familles (médicaux, psychologiques, relationnels, financiers, scolaires...), la multiplication des intervenants, la complexité croissante des situations rencontrées...

5 Dès lors que différents intervenants et institutions se trouvent sollicités par ces familles en détresse, la question de la coexistence et de la pluralité se pose. Elle se pose non seulement au sein de chacun des organismes pris séparément (hôpital, centre de santé mentale, école...). notamment parce qu'ils sont souvent constitués d'équipes pluridisciplinaires, mais aussi au niveau du réseau d'institutions, puisque les initiatives allant dans le sens d'une concertation, d'une collaboration, d'un partenariat entre les différents intervenants se multiplient.

6 A cette problématique de la coexistence est intimement liée, nous semble-t-il, celle de la pluralité de savoirs et de compétences, que l'on trouve au sein d'une organisation. Dans ce domaine psycho-médico-social. on peut en effet rencontrer des équipes pluridisciplinaires, avec des fonctions aussi variées que : médecin, psychologue, assistant social, infirmier, éducateur...

$7 \mathrm{Au}$ sein du réseau de professionnels mobilisé par une même famille, cette variété de spécialités et de disciplines peut se voir démultipliée : puéricultrice, conseiller d'aide à la jeunesse, médiateur de dettes, etc. peuvent être impliqués. Si associer des professions et des compétences différentes constitue en quelque sorte le propre de toute organisation, on retrouve cette caractéristique dans le réseau. Les questions relatives à la coexistence de savoirs multiples et à la place de chacun s'y posent donc avec beaucoup d'acuité, d'autant que l'ensemble des personnes concernées ne se retrouve pas sous la bannière d'une même institution : ces dernières tendent, au contraire, aussi à se multiplier.

Ceci nous amène à un second niveau auquel la coexistence peut être envisagée : celui de la culture d'organisation. Quand des activités de réseau (collaborations, partenariats, etc.) se multiplient, on peut considérer que ce sont, en plus des savoirs, des formes de cultures d'organisation diverses qui coexistent. Nous entendons par là « les habitudes de travail, les règles et nonnes informelles, les images collectives, les manières de percevoir et de comprendre collectives $»^{4}$. Celles-ci peuvent différer d'une institution à l'autre. Par exemple, il est possible que deux assistants sociaux, appartenant à des institutions différentes, (par exemple un hôpital et une pouponnière) travaillent auprès d'une même famille, règlent avec elle des questions différentes et envisagent leur travail et leurs rapports avec la famille de manière divergente.

9 Nous avons présenté différentes manières assez classiques d'envisager la problématique de la coexistence dans un réseau psycho-médico-social. Voyons maintenant comment l'apport de la théorie de la justification peut nous permettre d'affiner cette question. 


\section{Penser la coexistence à partir de la théorie de la justification}

10 La prise de décisions et la coordination de l'action collective sont, avec la détermination d'objectifs communs, quelques-uns des ingrédients nécessaires au bon fonctionnement d'une organisation. Or il existe de nombreux principes sur lesquels s'appuyer pour prendre une décision ou se coordonner. Thévenot dresse le portrait « d'une organisation composite qui repose sur une pluralité d'ordres de ressources. La mise en valeur de ces ressources relève d'une pluralité d'impératifs différents $»^{5}$. Boltanski et Thévenot ont réalisé une modélisation théorique de ces différents impératifs. Ils proposent des idéauxtypes de principes d'accord qui peuvent être mobilisés pour prendre une décision et la justifier, parvenir à un accord ou encore pour coordonner l'action collective.

11 En effet, "chaque impératif n'est pas seulement un principe mais aussi une façon de coordonner effectivement des actions collectives en fondant des formes communes de jugement ainsi que des procédures de mise à l'épreuve " ${ }^{6}$. c'est-à-dire un monde. Ainsi, les actions peuvent être justifiées et coordonnées en faisant référence à différents mondes : marchand, industriel, civique, domestique, inspiré, de l'opinion ou encore par projets 7 . Le principe de justification et de coordination peut relever plutôt d'une logique marchande économique, d'une logique d'efficacité industrielle, de représentation civique, de tradition et de loyauté domestique, d'inspiration authentique, de réputation ou encore d'une logique de projets.

12 L'intérêt principal de cette théorie de la justification est qu'elle l'ait droit à la pluralité et à la diversité qui peuvent s'y envisager en termes d'ordres de grandeur. Ceux-ci servent à établir la grandeur la valeur en quelque sorte d'un être - aussi bien d'une personne que d'un objet - en fonction d'un principe particulier, par exemple la valeur marchande, la renommée etc. Les ordres de grandeur diffèrent des valeurs, pour deux raisons : d'une part parce qu'ils s'appuient sur les objets présents dans la situation, d'autre part, parce que celte théorie est conçue de manière dynamique. Aucune grandeur n'est attachée de manière définitive à un être : elle est toujours susceptible d'évoluer et d'être remise en question.

13 Une telle conception de la grandeur relative des êtres et donc de la diversité a des répercussions sur la manière de penser la coexistence : puisque la différence devient en quelque sorte contingente, sans cesse mouvante, il faut aussi penser la coexistence de manière dynamique.

14 Pour ces auteurs, il est important d'intégrer une pluralité d'impératifs pour analyser les organisations: "Dès lors que nous cherchons à comprendre le fonctionnement d'organisations à partir des actions des personnes impliquées, dès lors que nous suivons les acteurs, nous les voyons traverser les cloisonnements correspondant aux distinctions précédentes et se soumettre successivement à des impératifs différents. $\|^{8}$

15 Ils expliquent que les acteurs sont amenés à mobiliser alternativement ces différents mondes en fonction des situations : «dans les sociétés complexes que nous étudions, les mêmes personnes sont amenées, dans le cours de leur existence, et dans des laps de temps parfois très courts, à passer d'une orientation à l'autre ${ }^{9}$ ». Thévenot va même plus loin, jusqu'à dire que cette capacité de passer d'un ordre de grandeur à un autre, est un signe de normalité : « Dans les cas où cette flexibilité fait défaut, les conduites portent à la 
critique, paraissent anormales, et peuvent même suggérer la folie $»^{10}$. C'est là la dimension dynamique de cette théorie.

Il n'est donc pas question ici de coexistence d'altérité radicale, vu que celle-ci est toujours considérée en situation et qu'un acteur peut, dans une situation, mobiliser divers ordres de grandeur.

Le rapport entre ces ordres de grandeur qui coexistent dans les organisations est critique, c'est-à-dire que "chaque ordre de grandeur contribue à réduire à l'insignifiant, au circonstanciel, voire au défectueux, ce qui importait dans l'autre $»^{11}$. En effet, dans leur théorie, rien - et surtout pas l'état d'une personne n'est jamais fixé définitivement : tout est toujours susceptible de remise en question, notamment par la mobilisation d'un autre ordre de grandeur: "Lorsqu'on est "grand» dans un monde, on est " petit» dans un autre : les êtres reconnaissants dans l'un n'ont plus d'existence dans un autre : le langage utilisé dans l'un n'est plus compris dans un autre. [Par conséquent] être engagé dans l'un puis dans l'autre, traduire les êtres de l'un à l'autre, agencer ensemble des êtres qui relèvent de plusieurs mondes d'action, sont des opérations coûteuses. $»^{12}$. Au fond, les auteurs soulignent que la coexistence a un prix qui n'est pas négligeable.

Suivre cette théorie a des implications en termes de positionnement épistémologique du chercheur par rapport à son objet, et donc par rapport à la question de la coexistence envisagée ici : « Dès lors que l'on atteste l'existence symétrique de cités différentes, ou de mondes d'action différents, on perd l'assurance critique de ceux qui croient à un seul monde, et donc à une réalité unique de base. Mais on en gagne une autre en donnant comme visée au travail sociologique de montrer les tensions qui résultent de la confrontation entre mondes. $»^{13}$. C'est d'ailleurs pour cette raison que cette théorie se révèle si intéressante pour étudier les conflits, les désaccords etc. où viennent souvent se cristalliser des questions qui ont trait à la coexistence.

Nous ne rentrerons pas ici davantage dans le détail de la théorie développée par ces auteurs. Nous nous en tiendrons à la mise en exergue du principe à la base de cette théorie et à la réflexion sur la manière dont il permet d'aborder la pluralité, la complexité et la coexistence. Pour une description détaillée des différents impératifs, que les auteurs conceptualisent sous le terme de mondes, nous renvoyons à leur ouvrage ${ }^{14}$. Nous n'avons pas non plus abordé ici l'ensemble des concepts développés dans le cadre de cette théorie, que ce soit les axiomes de base (notion de commune humanité, etc.) ou encore les différentes notions qui servent à dresser le portrait d'un monde: principe supérieur commun, état de grandeur, dignité. ${ }^{15}$. Notons qu'on en trouvera tout de même quelques traces dans le point suivant, qui expose un septième monde décrit par Boltanski et Chiapello. Celui-ci fait référence au projet et il nous semble intéressant pour la problématique du travail en réseau.

\section{Le monde par projet : un aspect de la théorie de la justification adapté au réseau?}

Après avoir mis en exergue la manière dont la théorie de la justification fait droit à la pluralité et permet de penser la coexistence. à partir d'une pluralité de mondes d'action, nous nous attarderons sur l'un d'eux en particulier: le monde par projets. Cette démarche se justifie de deux manières. D'une part, ce monde est défini par Boltanski et Chiapello en faisant référence au réseau: et comme nous nous intéressons à la 
problématique de la coexistence dans un réseau d'intervenants psychomédico-sociaux, il peut avoir un potentiel explicatif important pour notre objet. D'autre part, présenter un monde particulier constitue aussi une occasion de montrer comment se déploie cette théorie de la justification et comment les auteurs s'y prennent pour décrire un impératif particulier.

21 Étant donné qu'il s'agit d'une recherche en cours, il n'est pas possible au stade actuel de présenter des résultats d'analyse pour la partie empirique. Nous nous en tiendrons essentiellement à une présentation théorique du monde par projets, tout en indiquant ça et là des pistes de réflexion en rapport avec notre objet d'étude. Les idées avancées à ce niveau doivent être considérées à titre d'hypothèse, étant donné qu'un travail d'articulation plus précis entre théorie et pratique doit encore être effectué.

Comme toutes les autres, cette cité $^{16}$ propose de rendre saillants certains aspects de la réalité sociale, certains modes de raisonnement, de fonctionnement et en laisse d'autres dans l'ombre. Il ne faut donc pas perdre de vue la dimension de pluralité des mondes d'action présentée dans la partie précédente.

Dans le modèle de la cité par projets, la vie sociale est envisagée comme étant faite d'« une multiplication de rencontres et de connexions temporaires, mais réactivantes, à des groupes divers, opérées à des distances sociales, professionnelles, géographiques, culturelles éventuellement très grandes ${ }^{17}$. Le réseau serait donc un ensemble, un tissu de personnes, de groupes, d'entités diverses, voire disparates connectables les uns aux autres et le projet. «l'occasion et le prétexte de la connexion $»^{18}$. Ce dernier se définit partiellement en référence au réseau: "Celui-ci rassemble temporairement des personnes très disparates et se présente comme un bout de réseau fortement activé pendant une période relativement courte, mais qui permet de forger des liens plus durables qui seront ensuite mis en sommeil tout en restant disponibles $»^{19}$.

Dans le cadre de la prise en charge d'une famille dans le domaine psycho-médico-social, on pourrait envisager le fait de faire appel à tel ou tel intervenant pour «traiter" tel aspect de la problématique familiale, comme une l'orme de connexion entre certains membres du réseau, participant à la mise sur pied d'un projet plus global d'aide à la famille.

Dans le cadre d'un projet, tin certain nombre de personnes, de groupes ou d'entités sont activés, interconnectés, mobilisés et un ordre local peut se mettre en place.

Maintenant que nous avons expliqué le choix du terme " projet » et ses liens par rapport à la notion de réseau, nous pouvons vraiment définir ce qu'est la cité par projets, c'est-àdire : « un système de contraintes pesant sur un monde en réseau incitant à ne tisser des liens et à n'étendre ses ramifications qu'en respectant les maximes de l'action justifiable propres aux projets $»^{20}$.

27 Précisons que toutes les cités peuvent être considérées comme des systèmes de contraintes incitant à agir uniquement dans le respect de leur principe supérieur commun. Dans cette septième cité, les projets constituent des entraves au réseau et « à la circulation absolue » pour les raisons suivantes : «ils réclament un certain engagement, quoique temporaire et partiel, et supposent un contrôle, par les autres participants, des qualités que chacun met en œuvre $»^{21}$.

On pourrait s'interroger sur les formes que peuvent prendre cet engagement et ce contrôle intersubjectif des qualités et compétences que les différents intervenants 
psycho-médico-sociaux mettent en œuvre dans le réseau qui se constitue autour d'une famille.

Les différents termes de la grammaire ${ }^{22}$ de la cité par projets qui déterminent les maximes de l'action justifiable propres aux projets vont maintenant être développées en passant en revue les différentes notions caractéristiques d'une cité.

Ce qui compte dans ce inonde, le principe supérieur commun, c'est l'activité, qu'il s'agisse de travail salarié, bénévole, éducatif, associatif etc.., il faut être occupé. « avoir toujours quelque chose en vue, en préparation avec d'autres personnes que la volonté de faire quelque chose conduit à rencontrer $»^{23}$. On ne parle plus d'emploi mais de portefeuille d'activités que chacun gère. Celte activité vise, bien évidemment, à générer des projets. Dans cet esprit, développer au maximum son réseau de relations et faire proliférer les liens participent également de la grandeur de cette cité: «Le projet n'ayant pas d'existence hors de la rencontre (puisque, n'étant pas inscrit une fois pour toutes dans une institution ou un environnement, il se présente en action, à faire, et non sous la forme de ce qui serait déjà là) l'activité par excellence consiste à s'insérer dans des réseaux et à les explorer, pour rompre son isolement et avoir des chances de rencontrer des personnes ou de frayer avec des choses dont le rapprochement est susceptible d'engendrer un projet. L'activité se manifeste dans la multiplicité des projets de tous ordres. $»^{24}$

31 Est donc considéré comme grand dans ce monde, celui qui peut mettre en lien, qui est engageant - pour inciter à s'impliquer dans un projet -. flexible, mobile, autonome, à l'écoute, tolérant. Le grand sait repérer les bonnes sources d'informations, faire le tri entre les connexions riches en potentiel et les autres. Mais le grand ne doit pas se contenter d'identifier les connexions porteuses, il doit pouvoir établir des relations aussi durables qu'il lui semble nécessaire, ce qui implique ne pas repousser les gens, attirer leur sympathie, en un mot les « intéresser " notion que les auteurs reprennent à Callon ${ }^{25} \mathrm{Il}$ optimise sa ressource rare : le temps. Il a de l'intuition, du talent.

On suppose assez aisément que ce genre de qualités peut se révéler bien utile pour tout qui s'efforce de mettre en place des partenariats entre institutions, comme c'est le cas dans le domaine psycho-médico-social.

Le répertoire des sujets, en quelque sorte les êtres, les plus représentatifs de cette cité, sont le médiateur - au sens de celui qui « met en rapport ». " fait des liens ». " tisse des réseaux $»^{26}$ - le chef de projet, le coach, l'innovateur ou encore l'expert. Ils ont en commun de parvenir à frayer les liens les plus riches, cette richesse étant définie par la distance, que ce soit géographique, sociale, temporelle ou institutionnelle, qu'elle permet de franchir et « l'art de la conciliation des contraires $»^{27}$. Le grand qui parvient à établir ce genre de relation particulièrement riche se trouve donc, temporairement au moins, en position de passage obligé - concept également repris aux sociologues de l'innovation parce que les autres doivent passer par lui pour activer cette connexion.

Ils sont donc doués pour "réunir et mettre en communication des personnes très différentes ${ }^{28}$. Le grand a une stratégie de conduite des relations, c'est-à-dire qu'il possède une capacité à moduler et mettre en avant certains aspects de sa personnalité de nature à faciliter les contacts. Il sait aussi prêter attention aux autres et récolter des informations qui lui permettront de gérer les situations d'incertitude et d'v agir. Il possède également talent et intuition. S'il est créatif, sa créativité prend une forme 
collective, elle « est fonction du nombre et de la qualité des liens $»^{29}$ et s'exprime alors plutôt dans la recombinaison originale d'éléments.

Quant aux objets, ils sont aussi liés à l'activité de mise en relation, comme les nouvelles technologies de l'information et de la communication, et comprennent tous les instruments de connexion, y compris les relations informelles, de confiance, le partenariat sans oublier le projet en lui-même. « D'où l'importance accordée aux relations de l'ace à face, à la responsabilisation, à la confiance, aux situations vécues ensemble, à la parole donnée (qui vaut tous les contrats), à l'entraide à la coopération, dans l'établissement des partenariats, dans le montage des projets, dans la construction des réseaux (...). Ce qui importe le plus étant intangible, informel, impalpable, informel terme qui qualifie ici aussi bien les relations que les régies du jeu « qui s'inventent au fur et à mesure »- les dispositifs les plus idoines sont donc interpersonnels $»^{30}$.

Dans le domaine psycho-médico-social, il nous semble que l'organisation de rencontres de "coordination» entre divers intervenants autour de thématiques particulières (petite enfance, décrochage scolaire...) donne une bonne idée de l'importance accordée à ces éléments dans le réseau. Un des objectifs plus ou moins avoués est que les différents professionnels apprennent à mieux se connaître et développent des relations informelles: en outre, l'accent est souvent mis sur la convivialité dans ce genre de rencontres.

La forme de relation naturelle entre les êtres est la connexion, la rencontre. On en sort modifié, par l'échange d'informations qui a lieu. En effet, dans un réseau, la relation et l'information entretiennent des rapports particuliers. Tout d'abord, le capital relationnel et d'information $\mathrm{y}$ sont tous deux très importants. Ensuite, ils s'influencent mutuellement: comme le fonctionnement en réseau est basé sur des relations de confiance, l'information communiquée par un agent est ramenée par celui qui la reçoit à la représentation qu'il a de cette personne, pour être réinterprétée en fonction. En outre, l'information qui s'échange modifie la relation. L'information est à la fois le résultat et la condition de multiplication des connexions.

Dans la prise en charge d'une famille, cet échange d'informations entre intervenants peut revêtir une importance capitale: parfois, il en va de la sécurité de l'enfant. Mais des questions de respect du secret professionnel, que l'on sait chères aux travailleurs de ce secteur, y sont également mêlées. Cela touche également à l'établissement de relations de confiance, que ce soit avec la famille. - ce qui est crucial pour la relation thérapeutique ou entre professionnels. ce qui importe pour les questions de relais au niveau de la prise en charge.

La dignité, c'est-à-dire la caractéristique générale des êtres humains sur laquelle la cité par projets se base et qui garantit que tout un chacun peut être grand dans ce monde, est le besoin de se connecter, le désir d'être en relation.

Comme les projets sont par nature limités dans le temps, il importe de pouvoir passer de l'un à l'autre, rebondir à la fin d'un projet sur une autre initiative. Ceci constitue d'ailleurs l'épreuve modèle dans ce monde, c'est-à-dire une étape importante, un « test " qui permet de déterminer la grandeur d'un être. Le jugement s'exprime dans le l'ait d'être, à la fin d'un projet, appelé à participer à un autre projet, ou à en mettre un autre sur pied, ou encore dans le l'ail de vouloir être rencontré par d'autres, la sanction étant de se retrouver exclu, tenu à l'écart ; c'est le mode d'expression du jugement, C'est aussi la l'orme de l'évidence c'est-à-dire la modalité de connaissance propre à ce monde. 
41 On est par exemple petit si on est un piètre communicateur. si on n'est pas digne de confiance ou si on ne sait pas l'accorder, ou encore si on éprouve des difficultés à changer de projet. Les petits sont, par opposition aux grands, non flexibles, autoritaires, inengageables, peu mobiles, attachés à leur sécurité, etc.

42 Le type de relation qui les lie aux grands et qui fait que l'état de grand profite également aux petits, c'est-à-dire le rapport de grandeur, c'est la redistribution des connexions : en échange de leur engagement, le grand s'attache à augmenter l'employabilité des petits, à les faire en quelque sorte profiter de son réseau de relations.

43 Notons encore que, pour être considéré comme grand dans la cité pat-projets, il faut consentir les investissements suivants: l'adaptabilité, qui implique une certaine "légèreté, flexibilité », aussi bien spatiale, qu'en termes de valeurs - il importe d'être ouvert aux différences, de reconnaître les vertus de l'ambivalence, particulièrement en situations d'incertitude, de non attachement à la propriété, à la stabilité... L'homme connexionniste ne se laisse pas non plus « capter par les institutions, avec les obligations de toutes sortes que cela implique, ne se laisse pas enfermer dans des responsabilités envers les autres ou les organisations dont il aurait la charge $»^{31}$. Ceci pose évidemment la question de l'institution dans un réseau. Le sacrifice le plus fondamental que l'investissement dans la cité en réseaux peut impliquer est celui de sa personnalité, au sens où, à force de toujours devoir se façonner, s'adapter, se plier aux contraintes des situations et surtout des interlocuteurs qu'il rencontre, l'homme connexionniste peut en arriver à devoir faire le deuil d'une personnalité, c'est-à-dire d'un ensemble constant d'attitudes, de valeurs etc. au travers de toutes les situations. Il peut aussi être amené à sacrifier sur l'autel de la cité par projets les relations amicales, désintéressées par essence ou celles qui ne sont pas assez riches de potentialités. On perçoit donc là une possible dérive militariste.

44 Pour finir, la figure harmonieuse de l'ordre naturel est bien évidemment le réseau. Les auteurs expliquent également en quoi le projet est particulièrement bien adapté à la l'orme du réseau : "C'est précisément parce que le projet est une forme transitoire qu'il est ajusté à un monde en réseau : la succession des projets, en multipliant les connexions et en faisant proliférer les liens, a pour effet d'étendre les réseaux. ${ }^{32}$

Ils vont même plus loin, en proposant quelques considérations afférentes au réseau : son extension est considérée comme sa vie même, tandis que l'arrêt de celle-ci est assimilé à la mort, celle-ci pouvant également consister en « sa transformation en une organisation pyramidale $»^{33}$. La forme de la déchéance dans cette cité réside aussi dans la fermeture du réseau, qui peut alors se muer en petit groupe de gens qui se connaissent bien entre eux et s'échangent des privilèges.

46 Ceci incite évidemment aussi à réfléchir sur les réseaux psycho-médico-sociaux : sont-ils réellement ouverts à de nouveaux membres ou donne-t-on simplement le nom de réseau à des cliques de professionnels qui, au fil du temps, ont pris l'habitude de travailler ensemble?

47 Pour conclure cette partie sur la cité par projets, nous livrerons quelques-unes de nos hypothèses concernant l'articulation possible entre cette partie de la théorie de la justification et notre problématique de recherche. L'idée qui nous a guidée dans cette démarche est la suivante: la cité ${ }^{34}$ par projets, est particulièrement indiquée pour analyser le travail en réseau dans le domaine psycho-médico-social. Notre hypothèse est 
que, dans ce cas. le projet serait la détermination îles modalités de prise en charge ou d'un programme thérapeutique particulier pour la famille et avec elle.

Enfin, les choses sont plus complexes encore. On devrait alors plutôt considérer que, dans le cas de la prise en charge d'une famille, il y a un emboîtement de projets à différents niveaux du réseau psycho-médico-social. Chaque intervenant pris isolément peut avoir un projet vis-à-vis de la famille, les projets des différents intervenants avant à s'articuler les uns aux autres: il peut y avoir le projet d'une institution vis-à-vis de cette même famille, chaque institution ayant à la limite le sien : mais il pourrait aussi y avoir un projet partagé pur différentes institutions. - ou établi en concertation, par rapport à une situation familiale, et auxquels elles collaboreraient chacune. On peut encore imaginer que certaines institutions aient un projet ensemble, indépendamment du cas de la prise en charge d'une famille particulière. De manière générale, on peut penser qu'un projet peut être ce qui amène des gens d'horizons et d'institutions différents à coexister temporairement. Toutes ces idées sont avancées à titre d'hypothèses et devront encore être lestées dans une étape ultérieure de la recherche.

\section{Conclusion}

Nous avons vu que le problème de la coexistence dans un réseau psycho-médico-social peut être posé de différentes manières: premièrement, en termes de savoirs, de compétences et de spécialités, deuxièmement en termes de culture d'organisation, troisièmement, en termes d'ordre de grandeur, de principes ou d'impératifs généraux qui servent à justifier et à coordonner l'action. Lu différence entre les deux premières manières d'envisager la coexistence et la troisième tient au l'ait que dans les deux premières, la conception de la différence est plus «ontologique », définitivement fixée, alors que la troisième est dynamique. Nous voulons dire par là qu'un savoir ou une culture d'organisation semblent attachés à une personne de manière durable, même si elle peut en changer ou en posséder plusieurs. Tandis que la référence à un monde particulier est plus contextuelle, ponctuelle, voire changeante: selon Boltanski et Thévenot, nous devons tous pouvoir mobiliser des ordres de grandeur différents, en fonction de la situation, sous peine d'inadaptation sociale.

D'autre part, un des impératifs qu'ils proposent est basé sur la notion de projet, qu'ils relient au réseau. Nous avons proposé quelques hypothèses quant à l'intérêt de cet aspect de la théorie pour étudier la coexistence dans un réseau psycho-médico-social. Si on part de cette hypothèse selon laquelle, dans le domaine psycho-médico-social, les choses fonctionnent en réseau selon une logique de projet, il faut encore s'interroger sur les implications que cela peut avoir au niveau de la coexistence, en ternies relationnels (au niveau des rapports entre les gens) et cognitifs (au niveau des rapports entre les savoirs), C'est de cette manière que nous entendons poursuivre notre questionnement, à partir de nos matériaux de terrain. Pour conclure, ajoutons que les pistes lancées pour nos réseaux dans le domaine psycho-médico-social nous semblent pouvoir valoir potentiellement pour toute organisation en réseau, moyennant quelques adaptations. 
BIBLIOGRAPHIE

\section{BIBLIOGRAPHIE}

BOLTANSKI. L., THEVENOT, L., De la justification. Les économies de la grandeur, Paris : Gallimard. 1990.

BOLTANSKI. L., CHIAPELLO. E., Le nouvel esprit du capitalisme. Paris : Gallimard. 1999.

DODIER, N., Agir dans plusieurs mondes, in Critique.t XI.VII, n529-530, 1991. pp. 427-458.

HOTIER. H., La communication internationale des organisations à l'épreuve des cultures nationales et de la culture d'entreprise, in Communication.

THEVENOT, H., Tensions critiques et compromis entre définitions du bien commun : l'approche des organisations par la théorie de la justification, in AFFICHARD. J., Décentralisation des organisations et problèmes de coordination : les principaux cadres d'analyse, Paris : L'Harmattan, Institut International de Paris La Défense. 1997, pp. 93-115.

\& Organisation. $\mathrm{n}^{\circ} 10.2^{\mathrm{e}}$ semestre 1996. pp. 189-203.

\section{NOTES}

1. S agissant d'un travail de recherche doctorale en cours, l'accent sera davantage mis sur la problématisation et la formulation de questions soulevées par cette problématique.

2. Boltanski L., Thévenot L., De la justification. Les économies de lu grandeur. Paris : Gallimard. 1990.

3. Boltanski L., Chiapello L., Le nouvel esprit du capitalisme, Paris Gallimard. 1999.

4. Hotier H., La communication internationale des organisations a l'épreuve des cultures nationales et de la culture d'entreprise, in Communication \& Organisation. $\mathrm{n}^{\circ} 10.2 \mathrm{e}$ semestre $1996, \mathrm{p}$ 191.

5. Thévenot L., Tensions critiques et compromis entre définitions du bien commun : l'approche des organisations par la théorie de la justification In Affichard J. Décentralisation des organisations et problèmes de coordination: les principaux cadres d'analyse. Paris: L'Harmattan. Institut International de Paris La Défense. 1997. p. 97.

6. Thévenot L., op. cit., p. 95.

7. Pour une description détaillée de ces différents mondes, cf. Boltanski L., Thévenot L., op cit.. pp. 200-262.

8. Thévenot I... op cit., p. 98.

9. Idem, p. 98.

10. Idem, p. 98

11. Idem, p. 99.

12. Dodier N., Agir dans plusieurs mondes, in Critique, t XI VII. $\mathrm{n}^{\circ}$ 529-530. 1991. pp. 433-434.

13. Idem. p. 454.

14. Boltanski L., Thévenot L., op cit.

15. Pour une présentation plus détaillée, nous renvoyons à Boltanski L. et Chiapello E op. cit.. pp 163-165. 
16. De manière très résumée, on peut dire que ce terme désigne la forme idéal-typique d'un monde

17. Idem, p. 156

18. Idem, p. 157

19. Ibid.

20. Idem, p. 161

21. Ibid.

22. Les différents termes de cette grammaire ont été mis au point dans Boltanski L. et Thévenot L., op. cit., (cfr pp 163-165) Ils sont repris ici par Boltanski et Chiapello pour les appliquer à leur cité par projets

23. Boltanski L. et Chiapello L., op. cit., p. 166.

24. Ibid.

25. Idem, p. 170

26. Idem, p. 162

27. Idem, p. 173

28. Ibid.

29. Idem. p. 192

30. Idem, p. 178

31. Idem. p. 186

32. Idem, p. 167

33. Landier H. Vers l'entreprise intelligente. Paris. Calman-Lévy. 1991 cité dans Boltanski L. Chiapello E, op cit.. pp. 167-168.

34. De manière très résumée, on peut dire que ce tenue désigne la tonne ideal-typique du monde

\section{AUTEUR}

\section{ISABELLE DELIĖGE}

Université Catholique de Louvain. GreMS, LASCO 